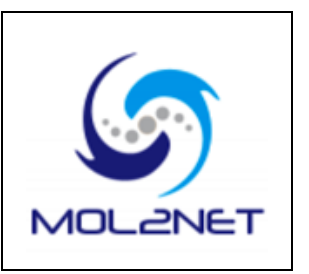

\title{
Fatty Acids Distribution Networks in Ruminal Membrane by Computational and Experimental Studies
}

\section{Yong Liu ${ }^{1,2,3 *}$, Zhiliang Tan ${ }^{2}$, Claudia Giovanna Peñuelas-Rivas ${ }^{1}$ and Esvieta Tenorio-Borroto ${ }^{1}$}

1 Faculty of Veterinary Medicine and Animal Science, Autonomous University of the State of Mexico, Toluca, 50090, México

2 Key Laboratory of Subtropical Agro-ecological Engineering, Institute of Subtropical Agriculture, the Chinese Academy of Sciences, Changsha, Hunan, 410125, P. R. China

3 Computer Science Faculty, University of A Coruña, Campus de Elviña s/n, A Coruña, 15071, Spain

* Yong Liu, e-mail: y.liu@udc.es. Published in Mol. BioSyst., 2015, Aug. DOI:

10.1039/C5MB00325C

Received: 6 October 2015 / Accepted: 8 October 2015 / Published: 2 December 2015

\begin{abstract}
The present communication introduces a new classification model for fatty acids (FA) distribution networks in ruminal microbe membrane based on experimental and computational studies. In the experimental part, long chain fatty acids and volatile fatty acids in ruminal microbe membrane or liquid phase were investigated by supplementation of different ratios of Omega-6 / Omega-3 and in the processes of base- / acid- methylation. In the computational part, Perturbation Theory (PT) and Linear Free-Energy Relationships (LFER), combined with corresponding Box-Jenkins $\left(\Delta \mathrm{V}_{\mathrm{kj}}\right)$ and PT Operators $\left(\Delta \Delta \mathrm{V}_{\mathrm{kj}}\right)$ were applied into the calculation of physicochemical parameters $\left(\mathrm{V}_{\mathrm{k}}\right)$ of fatty acids. The best PT-LFER model found to predict the effects of perturbations over the FA distribution network with Sensitivity, Specificity, and Accuracy $>80 \%$ for 407,655 cases. In final, PT-LFER model based on LDA was used to reconstruct the complex networks of perturbations in the FA distribution and compared with random Erdős-Rényi network models. The detail results have been published in Mol. BioSyst., 2015, Aug., the present is a short communications.
\end{abstract}

Keywords: Fatty acids, Distribution networks, Computational, Experimental, Ruminal membrane

\section{Introduction}

The $\omega-6 / \omega-3$ ratio plays an important role not only in the pathogenesis of cardiovascular diseases, but also in inflammatory, cancer and autoimmune diseases ${ }^{1-3}$. It is an efficient way to benefits the fatty acids composition in the diets by enrichment of ruminant meat or milk with $\omega-3$ polyunsaturated fatty acids (PUFAs). However, the ruminal complex biohydrogenation process limits their bioavailability of Omega- $3{ }^{4}$. On the other hand, methylation methods are directed 
http://sciforum.net/conference/mol2net-1

effected the experimental values of PUFAs or volatile fatty acids (VFAs) distribution ${ }^{5-7}$. In the view of biology, the structure properties of long chain fatty acids (LCFAs, especially the number, location or topology structure of double bonds) are highly related to the chronic disease. To address this problem, it was postulated that the LCFAs in ruminal microbial membranes change with the supply of $\omega-6 / \omega-3$ ratios.

Chemoinformatics is related to Chemometrics, data mining, Machine Learning ${ }^{8}$ accompany with Chemistry, Information Science, and the areas of topology, chemical graph theory. A new Corwin Hansch model is based on lipophilicity-activity relationship ${ }^{9}$.

$$
\mathrm{f}\left(\varepsilon_{\mathrm{i}}\right)=\mathrm{a}_{0}+\mathrm{a}_{1} \cdot \log \mathrm{P}_{\mathrm{i}}+\mathrm{a}_{2} \cdot \mathrm{pK_{ \textrm {a } }}+\mathrm{a}_{3} \cdot \mathrm{MR}-\mathrm{a}_{4} \cdot\left(\log \mathrm{P}_{\mathrm{i}}\right)^{2}
$$

Steric, electrostatic, and hydrophobicity factors combined with water/n-octanol partition coefficients $\left(\mathrm{P}_{\mathrm{i}}\right)$, molecular refractivity (MR), acidity constants logarithmic (pKa) ${ }^{10}$ etc. might be biologically relevant were set as input variables of model ${ }^{11,12}$. A molecular property $\left(\varepsilon_{i}\right)$ or a function of this property $f\left(\varepsilon_{i}\right)$ for a given chemical compound or molecular entity $\left(\mathrm{m}_{\mathrm{i}}\right)$ was set as output of the model.

Hansch model is an extra-thermodynamic approach closely related to LFER ${ }^{13,14}$. The input variables $\left({ }^{\mathrm{i}} \mathrm{V}_{\mathrm{k}}\right)$ can be calculated as physicochemical parameters or molecular descriptors. In fact, the basic assumption for

\section{Results and Discussion}

The imbalance intake of $\omega-6 / \omega-3$ has the potential to induce some chronic diseases, such as inflammation, asthma, vascular disease ${ }^{19}$. In this study, long chain fatty acids (LCFAs) in microbial membrane, volatile fatty acids (VFAs) in media were differentiated on the conditions of exogenous $\omega-6 / \omega-3$ ratios. First, cis-FA increased and trans-FAs decreased with exogenous $\omega-3$ PUFA in bacteria phase. This reflects that the ratio
Hansch's analysis is based on the theory of similar molecules with similar activities. ${ }^{15-17}$ In addition, the "small" variations or perturbations at the molecular structural level need to be quantified.

The Perturbation Theory (PT) ${ }^{18}$ can be used to account for "small" problem by the view of Chemoinformatics. In this work, PT and LFER ideas were used to formulate a new PT-LFER approach for complex networks of FA distribution in Lipidomics. In this work, the first experiments were carried out to determine LCFA compositions in the rumen microbiome by addition of different ratios of omega-3 / omega- 6 . Then, Chemoinformatics study was provided, and the validation of new PT-LFER classification models. Artificial Neural Networks (ANNs) were used to test PT-NLFER models (compared to NonLinear). Next, the best PT-LFER model found was used to predict the effect of perturbations on initial boundary conditions over a large complex network of FA distribution/uptake in the ruminal microbiome. After that, the observed complex network was constructed and compared to the predicted network and random networks model with similar scale for the first time.

In a word, the present work paves the way to combine the perturbation theory with complex fatty acids molecular systems under the consideration of chemical structures and various boundary experimental conditions.

of cis/trans-FAs increased with the exogenous $\omega$ 3 PUFA ratios. That means exogenous PUFAs are degraded by rumen microorganisms to some extent, or have more complex metabolism processes to intermediary metabolism in both of cis- and trans- unsaturated FAs formulation. This study showed that $\omega$-3 PUFA ( $\alpha$-linolenic acid) could increase the cis-FAs content compared to $\omega$ 6 PUFA (linoleic acid) on both of bacteria and 
protozoa phases. The biohydrogenation of linoleic acid (cis 9, cis 12- C18:2) is first isomerized to conjugated linoleic acid (CLA, cis 9, trans 11$\mathrm{C} 18: 2$ isomer) in rumen environment, then conversion to vaccenic acid (trans 11- C18:1), or further reduction to stearic acid (saturated C18:0) 20. Whereas the bio-hydrogenation of $\alpha$-linolenic acid (ALA) in rumen is first characterized by isomerization to isomer $(9,11,15$ - cis, trans, cis$\mathrm{C} 18: 3$ ) and subsequent reduction by isomerase and/or reductase via cis, trans- isomers of C18:2, C18:1 and in final to stearic acid ${ }^{21}$.

A new model was developed powerful to predict FAs distribution networks in various phases of ruminal microbiome in addition of/out of exogenous PUFAs after perturbation dealing within chemical molecular descriptors $\left(\mathrm{V}_{\mathrm{k}}\right)$ and initial experimental boundary conditions $\left(\mathrm{c}_{\mathrm{j}}\right)$.
Each ' $f\left(\mathrm{~L}_{\mathrm{nr}}\right)_{\text {new }}$ represents a corresponding coefficient in the new model for predicting IPA(\%)new. This model can classify as high $\left(\mathrm{L}_{\mathrm{nr}}=\right.$ $1) /$ low $\left(\mathrm{Lnr}_{n r}=0\right)$ of the expected values of FAs (LCFAs/VFAs) between the new and reference states after changed the boundary conditions $\left(\mathrm{c}_{\mathrm{j}}\right)$. The parameter $n\left(\mathrm{Lnr}_{\mathrm{n}}=1\right)$ represents the number of cases in the sub-set with $\mathrm{Lnn}_{\mathrm{nr}}=1$ (links in complex network), or means the IPA(\%)new of new sub-set is higher than that of reference (IPA(\%)ref). Meanwhile, $n\left(\mathrm{~L}_{n r}=0\right)$ represents the number of cases observed and predicted in the sub-set with $\mathrm{L}_{\mathrm{nr}}=0$ (without connected nodes in complex networks) or implied that IPA(\%) $\%$ new is lower than IPA $(\%)_{\text {ref. }}$ The best PT-LFER model found using the LDA algorithm theory has only 12 independent variables and presented as following algorithm.

$$
\begin{gathered}
{ }^{\prime} f\left(L_{n r}\right)_{\text {new }}=-0.021 \cdot f\left(\varepsilon_{i j}\right)_{r e f}+0.0026 \cdot\left\langle f\left(\varepsilon_{i j}\right)\right\rangle_{\text {ref }}+0.3713 \cdot{ }^{\text {new }} V_{6}+1.0709 \cdot{ }^{\text {new }} \omega 6-1.1264 \cdot{ }^{\text {new }} \omega 3 \\
+0.0237 \cdot \Delta \Delta V_{5}\left(c_{1}\right)-0.0063 \cdot \Delta \Delta V_{6}\left(c_{2}\right)+0.0044 \cdot \Delta \Delta V_{1}\left(c_{4}\right)-0.0037 \cdot \Delta \Delta V_{1}\left(c_{5}\right) \\
-0.0036 \cdot \Delta \Delta V_{4}\left(c_{6}\right)-0.1682 \cdot\left({ }^{\text {new }} V_{6}\right)^{2}+0.0182 \cdot\left(\Delta \Delta V_{6}\left(c_{3}\right)\right)^{2}-13.7236 \\
N=407,655 ; \quad \chi^{2}=244,532.9 ; \quad p<0.005
\end{gathered}
$$

The output function ' $f\left(\mathrm{~L}_{\mathrm{nr}}\right)_{\text {new }}$ is useful to classify the pairs of states (pairs of nodes). Some input terms were expanded as follows. Like, $\Delta \Delta \mathrm{V}_{\mathrm{k}}\left(\mathrm{c}_{\mathrm{j}}\right)=$ $p\left(\mathrm{c}_{\mathrm{j}}\right)_{\text {new }} \cdot \Delta \mathrm{V}_{\mathrm{k}}\left(\mathrm{c}_{\mathrm{j}}\right)_{\text {new }}-p\left(\mathrm{c}_{\mathrm{j}}\right)_{\text {ref }} \cdot \Delta \mathrm{V}_{\mathrm{k}}\left(\mathrm{c}_{\mathrm{j}}\right)_{\text {ref. }}$ This can be further expanded in turn as $\Delta \Delta \mathrm{V}_{\mathrm{k}}\left(\mathrm{c}_{\mathrm{j}}\right)=\mathrm{p}\left(\mathrm{c}_{\mathrm{j}}\right)_{\text {new }}$ $\left({ }^{\text {new }} \mathrm{V}_{\mathrm{k}}-<\mathrm{V}_{\mathrm{k}}\left(\mathrm{c}_{\mathrm{j}}\right)>_{\text {new }}\right)-\mathrm{p}\left(\mathrm{c}_{\mathrm{j}}\right)_{\text {ref }}\left({ }^{\mathrm{ref}} \mathrm{V}_{\mathrm{k}}-<\mathrm{V}_{\mathrm{k}}\left(\mathrm{c}_{\mathrm{j}}\right)>_{\text {ref }}\right)$, $\left.<\mathrm{V}_{\mathrm{k}}\left(\mathrm{c}_{\mathrm{j}}\right)\right\rangle$ is the average of $\mathrm{V}_{\mathrm{k}}$ for each $\mathrm{c}_{\mathrm{j}}$. The statistical parameters, such as specificity (Sp), sensitivity ( $\mathrm{Sn}$ ), and accuracy (Ac) are always used to evaluate a new model. For the present study, the best new model found predicted the effects of perturbations under the initial conditions $\left(\mathrm{c}_{\mathrm{j}}\right)$ over FA distribution with $\mathrm{Sn}, \mathrm{Sp}$, and Ac greater than $80 \%$ for a total of $=407,655$ cases in training and external validation series.

Additional machine learning techniques were used to do some artificial neural networks - linear and non-linear ANNs (LNNs and MLPs) for comparing them with LDA model found. The best 11 ANN models were found to compare with the best LDA classification. In present study, the LNN models are based on 8 to 12 variables and MLP models have 5 to 12 input variables. The best MLP model (MLP 12:12-11-1:1) has 12 input variables and only one hidden layer with 11 neurons with $93.73 \%$ of accuracy in training set and $92.54 \%$ in validation set. The PT-NLFER model obtained with MLP number 6 classified our dataset better than the LDA PT-LFER model. However, PT-LFER is notably simpler and shows a direct relationship between the input variables and the output. Thus, the LDA model has a better prediction capacity than all LNNs but less than MLPs. In addition, LDA model has the lower training and validation errors compared to all ANNs. In a word, MLP models were better 
http://sciforum.net/conference/mol2net-1

problem solving capacity, but notably more complicated.

Network biology ${ }^{22}$ is a very useful approach to shed light on the functional organization of the cell. Thus, the observed complex networks were built for perturbations in FA metabolism/distribution between ruminal media and microbial membrane. Two states are connected $\left(\mathrm{Lnr}_{\mathrm{nr}}=1\right)$ for both IPA( $(\%)_{\text {obs }}\left(f\left(\varepsilon_{\mathrm{ij}}\right)_{\text {new }}\right)$ and IPA( $(\%)_{\text {ref }}\left(f\left(\varepsilon_{\mathrm{ij}}\right)_{\mathrm{ref}}\right)$, if IPA(\%)obs - IPA(\%)ref $>$ 0 , and $\mathrm{L}_{\mathrm{nr}}=0$ otherwise. It was considered that $\mathrm{L}_{\mathrm{nr}}$ $=1$ (lined nodes) if both values of ' $f\left(\varepsilon_{\mathrm{ij}}\right)_{\text {new }}$ and

\section{Conclusions}

Combined with experimental and computational methodology are useful to study the effect of multiple conditional factors over fatty acids distribution networks on ruminal microbiome and liquid phase. Meanwhile, PT and LFER ideas can be combined to develop a PT-LFER model on fatty acid distribution network. PT Operators and Box-Jenkins of physicochemical parameters are

\section{Conflicts of Interest}

The authors declare no conflict of interest.

\section{References and Notes}

1. S. L. Kronberg, E. J. Scholljegerdes, G. Barcelo-Coblijn and E. J. Murphy, Lipids, 2007, 42, 1105-1111.

2. K. i. Ichihara and Y. Fukubayashi, Journal of Lipid Research, 2010, 51, 635-640.

3. J. G. Kramer, V. Fellner, M. R. Dugan, F. Sauer, M. Mossoba and M. Yurawecz, Lipids, 1997, 32, 1219-1228.

4. C. Hansch, A. R. Steward and J. Iwasa, Molecular Pharmacology, 1965, 1, 87-92.

5. C. Hansch, W. E. Steinmetz, A. J. Leo, S. B. Mekapati, A. Kurup and D. Hoekman, J Chem Inf Comput Sci, 2003, 43, 120-125.

6. H. Gonzalez-Diaz, S. Arrasate, A. GomezSanJuan, N. Sotomayor, E. Lete, L. Besada-
' $f\left(\varepsilon_{\mathrm{ij}}\right)$ ref predicted by new model have the probability $p\left(\mathrm{c}_{\mathrm{ij}}\right)>0.5$ with $f\left(\varepsilon_{\mathrm{ij}}\right)_{\text {ref }}=\operatorname{IPA}(\%)_{\text {obs }}$ $\operatorname{IPA}(\%)_{\text {ref }}>0$.

Last, two random networks models (random network 1 and 2) were built. To set each model with a number of nodes and links as similar as possible to the observed and predicted networks, respectively. The results showed that the average values of the topological distance, node degree and closeness are similar between the observed and predicted networks (1.83 vs. 1.77, $72.75 \mathrm{vs}$. 80.29 , and 0.000755 vs. 0.000836 , respectively).

useful to define some inputs. ANN algorithms are also valuable to test the performance of alternative PT-NLFER; Non-Linear models. In final, ER random network models can be carried out the comparative studies with the observed and predicted networks referred to the effect of perturbations on the fatty acid distribution processes.

Porto and J. M. Ruso, Current topics in medicinal chemistry, 2013, 13, 1713-1741.

(C) 2015 by the authors; licensee MDPI, Basel, Switzerland. This article is an open access article distributed under the terms and conditions defined by MDPI AG, the publisher of the Sciforum.net platform. Sciforum papers authors the copyright to their scholarly works. Hence, by submitting a paper to this conference, you retain the copyright, but you grant MDPI AG the non-exclusive and un-revocable license right to publish this paper online on the Sciforum.net platform. This means you can easily submit your paper to any scientific journal at a later stage and transfer the copyright to its publisher (if required by that publisher). (http://sciforum.net/about ). 